\title{
Activated Akt signaling pathway in invasive ductal carcinoma of the breast: Correlation with HER2 overexpression
}

\author{
SUNG-SHIN PARK ${ }^{1}$ and SUNG-WON KIM ${ }^{2}$ \\ ${ }^{1}$ Department of Pathology, Dongguk University International Hospital, Dongguk; \\ ${ }^{2}$ Department of Surgery, Seoul National University College of Medicine, Seoul, Korea
}

Received January 15, 2007; Accepted April 2, 2007

\begin{abstract}
Akt} / \mathrm{PKB}$ is a serine/threonine kinase that plays a crucial role in cell survival and apoptosis. Aberrant activation of pAkt is associated with various malignant human cancers, including breast carcinoma. In vitro studies show that pAkt activation is mediated by estrogen and acts as a downstream effector of HER2 with implications in breast cancer progression and drug resistance. We investigated the incidence of Akt activation in invasive ductal carcinoma and its correlation with other clinicopathological variables. Using tissue microarray technology, immunohistochemical expression of phosphorylated Akt (pAkt) at Ser-473 was evaluated in 127 cases of invasive ductal carcinomas, together with hormone receptors, HER2, p53, Ki-67 and other clinicopathological variables. Both nuclear and cytoplasmic expression was noted for pAkt, with 46 cases $(36.2 \%)$ showing high cytoplasmic pAkt expression and 37 cases (29.1\%) showing high nuclear pAkt expression. There was a significant association between both high cytoplasmic and nuclear pAkt expression with HER2 overexpression (both $\mathrm{p}<0.0001)$. There was also a positive correlation between high nuclear pAkt expression with both estrogen receptor and progesterone receptor status $(\mathrm{p}=0.042$ and $\mathrm{p}=0.015$, respectively). High cytoplasmic pAkt expression was associated with high Ki-67 expression ( $\mathrm{p}=0.052)$, however, there was no association between pAkt and p53 expression. In the present study, activation of the Akt pathway shows strong association with HER2 overexpression, which is consistent with many in vitro studies. Our study also showed a positive correlation between pAkt and hormone receptors, which suggested the possible mechanism of endocrine resistance in ER-positive breast cancer. These results also suggest the prognostic value of pAkt and its importance in the prediction of therapeutic response in invasive ductal carcinoma of the breast.
\end{abstract}

Correspondence to: Dr Sung-Won Kim, Department of Surgery, Seoul National University Bundang Hospital, 300 Gumi-dong, Bundang-gu, Seongnam-Si, Gyeonggi-do 463-707, Korea

E-mail: brca@korea.com

Key words: pAkt, HER2, breast, ductal carcinoma

\section{Introduction}

Akt, also known as protein kinase B, consists of a family of highly conserved serine/threonine kinases. After stimulation with growth factors and cytokines, PI3 kinase is activated and recruits Akt to the plasma membrane where it is phosphorylated. Phosphorylation of both threonine and serine residues fully activates Akt, which provides a survival signal that protects cells from apoptosis and mediates growth factor-induced cell proliferation. The Akt signaling pathway plays a critical role in controlling the balance between cell survival and apoptosis, and the disruption of normal Akt signaling frequently occurs in several human cancers (1).

The ErbB family of transmembrane receptor tyrosine kinases [ErbB1 or epidermal growth factor receptor (EGFR), ErbB2 or HER2/c-neu, ErbB3 or HER3, and ErbB4 or HER4] and ligands have been consistently implicated in mammary gland tumorigenesis (2). The HER2 proto-oncogene encodes a tyrosine kinase receptor that is amplified and overexpressed in a significant proportion of human breast tumors (3). Although no soluble ligand for HER2 is known, HER2containing heterodimers are potent activators of multiple signaling pathways, including the PI3K/Akt pathway (4).

Recent studies suggest that breast cancer exhibits increased activation of pAkt which is associated with HER2 overexpression and poor prognosis. This is supported by previous evidence that tumor cells overexpressing HER2 exhibit constitutive Akt activity (4). In addition, constitutive and inducible Akt activity promotes resistance to chemotherapy, trastuzumab or tamoxifen in breast cancer cells (5).

In this study we investigated the incidence of Akt activation in invasive ductal carcinoma through the immunohistochemical method and its correlation with HER2, p53, Ki-67, steroid receptors, as well as other conventional clinicopathological parameters.

\section{Materials and methods}

Specimens. The files and formalin-fixed paraffin-embedded tissue blocks of 127 surgically resected breast invasive ductal carcinoma cases examined at the Department of Pathology, Seoul National University Boramae Hospital from 1997 to 2003 were studied. Patient age, tumor size, nuclear grade, histologic grade and lymph node status were evaluated by a review of medical charts and pathologic records. 


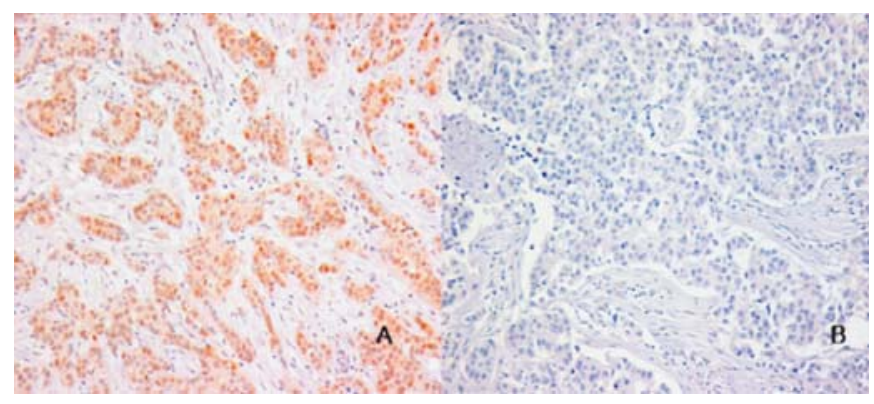

Figure 1. Representative immunohistochemical staining of pAkt. (A) Breast carcinoma showing cytoplasmic and nuclear expression of pAkt (original magnification, x200). (B) Negative immunohistochemical staining of pAkt (original magnification, $\mathrm{x} 200$ ).

Tissue microarray construction. Core tissue biopsies $(2 \mathrm{~mm}$ in diameter) were taken from individual paraffin-embedded breast carcinomas and the corresponding normal breast tissues, if possible (donor blocks), and arranged in a new recipient paraffin block (tissue array block) using a trephine apparatus (Superbiochips Laboratories, Seoul, Korea). Each tissue array block contained up to 60 cases, allowing four array blocks to contain the total of 127 cases. An adequate case was defined as a tumor occupying $>10 \%$ of the core area.

Immunohistochemistry. Sections $(2-\mu \mathrm{m})$ cut on silane-coated slides were deparaffinized with xylene and alcohol and subjected to antigen retrieval with a microwave in citrate buffer ( $\mathrm{pH}$ 6.0). Slides were also preincubated with a $2.5 \%$ hydrogen peroxide/methanol buffer and then with $0.3 \%$ bovine serum albumin/Tris-buffered saline blocking solutions. Primary antibodies used were pAkt (Cell Signaling, Beverly, $\mathrm{MA}$ ), estrogen receptor (ER), progesterone receptor (PR), Ki-67 (Dako Corporation, Carpinteria, CA), HER-2/neu (Scytek, West Logan, UT), and p53 (DO-7; Novocastra, Newcastle upon Tyne, UK). Primary antibody for pAkt was incubated at $4{ }^{\circ} \mathrm{C}$ overnight and other antibodies were incubated at room temperature for $1 \mathrm{~h}$ and were detected using anti-mouse (rabbit), horseradish peroxide-labeled polymer secondary antibody from Dako Envision (Dako Corporation). Staining was visualized with 3,3'-diaminobenzidine and counter-stained with hematoxylin.

Evaluation of immunostaining. Immunostaining was evaluated without the knowledge of clinical and pathological parameters. For pAkt, a semiquantitative assessment of both the intensity of staining and the percentage of positive cells was used. For the intensity, a score of 0-3 corresponding to negative, weak, moderate and strong positivity was recorded. In addition, the percentage of positive cells at each intensity was estimated. The total score was calculated as $(1 \mathrm{x}$ weak $\%$ $+2 \times$ moderate $\%+3 \times$ strong stained $\%)$. The range of possible scores was 0-300. Two cores were evaluated from each tumor. Each core was scored individually, and then the mean of the two readings was calculated. Only the invasive carcinoma was assessed for staining. The ER and PR were considered positive if $10 \%$ or more of the nuclei in the tumor were stained. HER-2/neu was scored by widely accepted criteria that assess the intensity and completeness of membrane staining; the staining intensity was graded on a scale from 0 to $3+(0$, negative; $1+$, weak; $2+$, moderate; and $3+$, intense), and scores of $0-1+$ were considered negative and scores of 2-3+ were positive. Staining for p53 was positive if $\geq 25 \%$ of nuclei were stained, and staining for Ki-67 was positive if $\geq 10 \%$ of nuclei were stained.

Statistical analysis. All statistical analyses were conducted using the SPSS 12.0 statistical software program (SPSS, Chicago, IL). Correlation between markers and with clinicopathological parameters was determined using Chi-square analysis. The results were considered to be statistically significant at $\mathrm{p}<0.05$.

\section{Results}

Expression levels of pAkt in primary breast cancer tissues. Akt is activated by phospholipid binding and by activation loop phosphorylation at threonine 308 by PDK1, and by phosphorylation within the carboxy-terminus at serine 473 . The phosphor-Akt (Ser473) antibody used in the present study detects Akt1 only when phosphorylated at serine 473, and Akt2 and Akt3 only when phosphorylated at equivalent sites. In our study pAkt expression was confined to the invasive tumor components, with no staining in normal breast epithelial cells. Both nuclear and cytoplasmic expression was noted for pAkt (Fig. 1) and semiquantitative assessment was conducted respectively. For subsequent analyses, the nuclear and cytoplasmic expression was categorized as high and low with a median cutoff value of 100 . Thirty-seven (29.1\%) cases showed a high nuclear pAkt score and 46 (36.2\%) cases showed a high cytoplasmic pAkt score. There was a positive correlation between cytoplasmic and nuclear expression $(\mathrm{p}<0.0001)$.

Relationship between pAkt and clinicopathological variables. The relationship between cytoplasmic and nuclear pAkt and the clinicopathological variables of the 127 patients is summarized in Tables I and II. High levels of cytoplasmic and nuclear pAkt were significantly associated with HER2 overexpression (both $\mathrm{p}<0.0001$ ). High levels of nuclear $\mathrm{pAkt}$ were also associated with high levels of nuclear ER and PR ( $\mathrm{p}=0.042$ and $\mathrm{p}=0.015$, respectively). But no significant correlation was observed between high cytoplasmic pAkt and hormone receptors. Among the other immunohistochemicalstaining variables, only Ki-67 showed a positive correlation with high cytoplasmic pAkt ( $\mathrm{p}=0.052)$. Also, high cytoplasmic pAkt showed statistically significant correlation with a younger age $(p=0.01)$. Although there was a weak relation between tumor size and pAkt status $(\mathrm{p}=0.094$ and $\mathrm{p}=0.088$, respectively), there was no significant correlation with other clinicopathological variables.

\section{Discussion}

Akt (protein kinase B) is a family of serine/threonine protein kinases with three mammalian isoforms (Akt1, Akt2 and Akt3), activated by growth factors in a PI3K-dependent manner at the plasma membrane where it is phosphorylated at Ser473 and Thr308 and becomes highly active $(6,7)$. 
Table I. Relationship between cytoplasmic pAkt expression and clinicopathological variables.

\begin{tabular}{|c|c|c|c|c|}
\hline \multirow[t]{2}{*}{ Characteristics } & \multicolumn{2}{|c|}{ pAKT score } & \multirow[t]{2}{*}{$\mathrm{p}$ value } & \multirow[t]{2}{*}{ Patients n (\%) } \\
\hline & Low $(\%)$ & High (\%) & & \\
\hline Total cases & $81(63.8)$ & $46(36.2)$ & & 127 \\
\hline \multicolumn{5}{|c|}{ Age at diagnosis (years) } \\
\hline$\leq 50$ & $30(37.0)$ & $28(60.9)$ & 0.0100 & $58(45.7)$ \\
\hline$>50$ & $51(63.0)$ & $18(39.1)$ & & $69(54.3)$ \\
\hline \multicolumn{5}{|l|}{ Tumor size (cm) } \\
\hline$\leq 2$ & $20(24.7)$ & $18(39.1)$ & 0.0880 & $38(29.9)$ \\
\hline$>2$ & $61(75.3)$ & $28(60.9)$ & & $89(70.1)$ \\
\hline \multicolumn{5}{|l|}{ Lymph node status } \\
\hline Negative & $33(47.1)$ & $20(50.0)$ & 0.7730 & $53(48.2)$ \\
\hline Positive & $37(52.9)$ & $20(50.0)$ & & $57(51.8)$ \\
\hline \multicolumn{5}{|l|}{ Nuclear grade } \\
\hline Low & $43(53.1)$ & $23(50.0)$ & 0.7380 & $66(52.0)$ \\
\hline High & $38(46.9)$ & $23(50.0)$ & & $61(48.0)$ \\
\hline \multicolumn{5}{|l|}{ Histologic grade } \\
\hline I and II & $40(49.4)$ & $25(54.3)$ & 0.5910 & $65(51.2)$ \\
\hline III & $41(50.6)$ & $21(45.7)$ & & $62(48.8)$ \\
\hline \multicolumn{5}{|l|}{ ER } \\
\hline Negative $(<10 \%)$ & $40(49.4)$ & $19(41.3)$ & 0.3800 & $59(46.5)$ \\
\hline Positive ( $\geq 10 \%)$ & $41(50.6)$ & $27(58.7)$ & & $68(53.5)$ \\
\hline \multicolumn{5}{|l|}{ PR } \\
\hline Negative $(<10 \%)$ & $37(45.7)$ & $15(32.6)$ & 0.1500 & $52(40.9)$ \\
\hline Positive ( $\geq 10 \%)$ & $44(54.3)$ & $31(67.4)$ & & $75(59.1)$ \\
\hline \multicolumn{5}{|l|}{ HER2 } \\
\hline Negative $(0 / 1+)$ & $60(74.0)$ & $15(32.6)$ & $<0.0001$ & $75(59.0)$ \\
\hline Positive $(2+/ 3+)$ & $21(26.0)$ & $31(67.4)$ & & $52(41.0)$ \\
\hline \multicolumn{5}{|l|}{ P53 } \\
\hline Negative $(<25 \%)$ & $47(58.0)$ & $30(65.0)$ & 0.4250 & 77 (60.6) \\
\hline Positive ( $\geq 25 \%)$ & $34(42.0)$ & $16(35.0)$ & & $50(39.4)$ \\
\hline \multicolumn{5}{|l|}{ Ki-67 } \\
\hline Negative $(<10 \%)$ & $53(65.4)$ & $22(47.8)$ & 0.0520 & $75(59.1)$ \\
\hline Positive ( $\geq 10 \%)$ & $28(34.6)$ & $24(52.2)$ & & $52(40.9)$ \\
\hline
\end{tabular}

Following activation Akt detaches from the membrane, which in turn, enables it to phosphorylate its cytosolic, as well as nuclear substrates, suggesting that compartmentalization of activated Akt may be important in determining its effects $(8,9)$. In the present study we observed both cytoplasmic and nuclear expression of pAkt; 36.2 and $29.1 \%$ respectively, which links this study to earlier in vitro studies suggesting that a fraction of activated Akt is translocated to the nucleus $(8,9)$.

Because the phospho-specific pan-Akt antibody used in our study recognized equivalent phosphorylation sites in Akt1, Akt2 and Akt3, we acknowledge that our positivestained paraffin-embedded specimens may have exhibited activation of any of the Akt isoforms expressed in that tumor, even multiple ones, due to the shared upstream signaling via $\mathrm{PI} 3 \mathrm{~K}$, and previous studies have shown that activated Akt is a more discriminatory prognostic factor than Akt1, Akt2 or Akt3 levels respectively (10-12).

HER2 is a member of the receptor tyrosine kinases, and is associated with several types of human cancer. Numerous studies have shown HER2 overexpression in $~ 30 \%$ of primary breast cancers and it is generally associated with poor prognosis. One of the major signaling pathways utilized by HER2 is the PI3K/Akt pathway. In vitro studies show that when HER2 is overexpressed, it forms heterodimers with HER3, which is the major docking site for PI3K. These HER2/HER3 dimers strongly activate the PI3K/Akt pathway to promote cell survival and tumor aggressiveness. In the present study, we found that pAkt expression correlated significantly with HER 2 overexpression $(\mathrm{p}<0.001)$, which is consistent with many in vitro studies using established cell lines and breast cancer tissues $(4,10,12)$. 
Table II. Relationship between nuclear pAkt expression and clinicopathological variables.

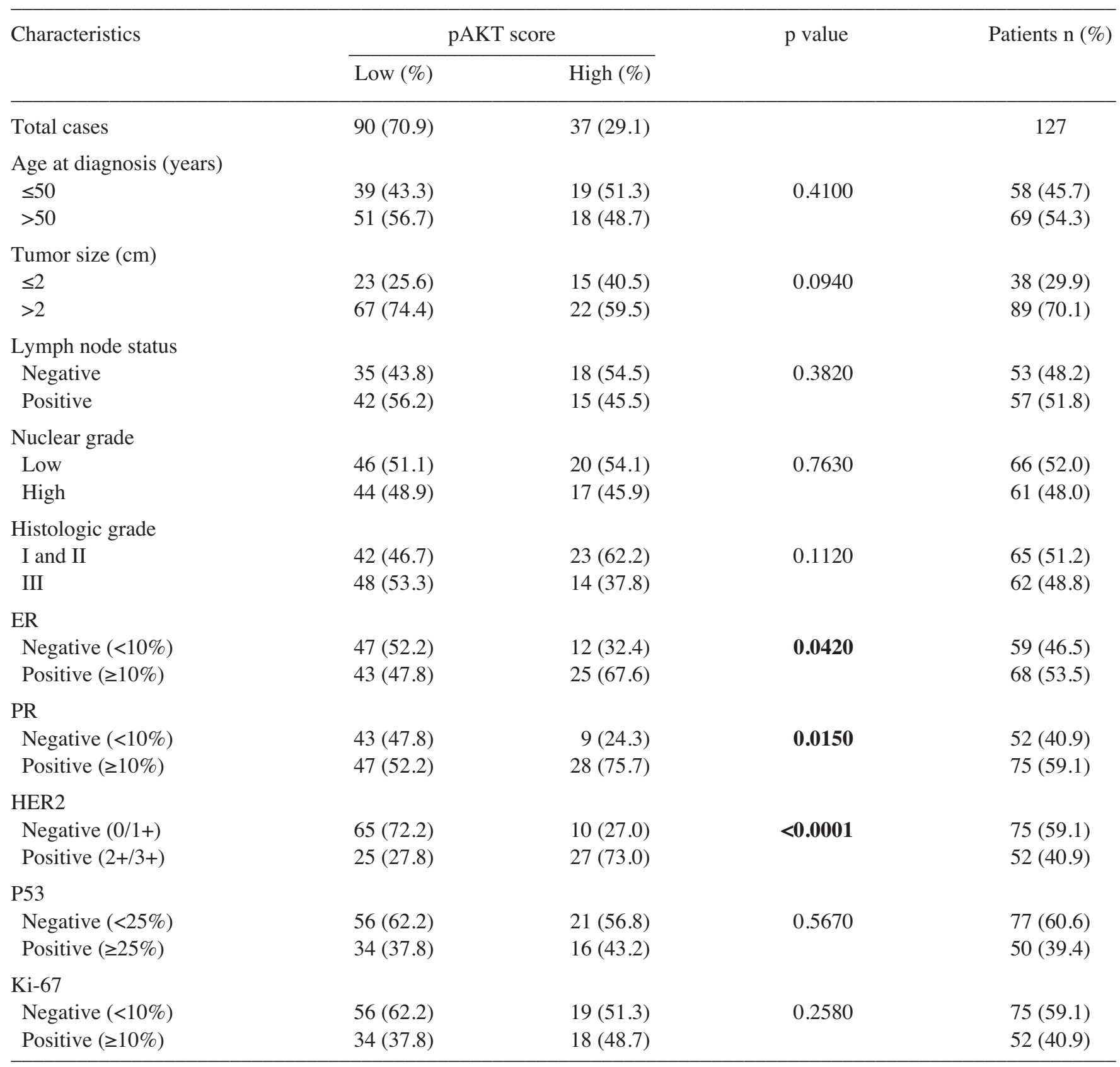

In addition, HER2 overexpression is associated with resistance to systemic and local radiation therapies. HER2 is strongly associated with activation of Akt in breast cancer cell lines and in tamoxifen-resistant MCF-7 cells $(13,14)$. The HER2/PI3K/Akt pathway plays a causal role in conferring a broad chemoresistance in breast cancer and justifies a strategy for targeting this pathway for enhancing breast cancer chemotherapy (15). These data, together with our findings, suggest that pAkt expression correlates with a potentially important prognostic marker for breast cancer, and that HER2 overexpression and Akt activation may result in a hormoneindependent phenotype and resistance to anti-hormone therapy such as tamoxifen.

Our study showed that high nuclear pAkt activity is closely related to high ER and PR status correlating with recent studies that provide evidence linking the activation of
Akt with that of HER2 and ER (16). Our study also supports the existing pre-clinical data of cross-talk between pAkt, HER2 and ER in resistance to endocrine therapy $(17,18)$. However, unlike nuclear pAkt, cytoplasmic pAkt showed no correlation with hormone receptors. Previous immunohistochemical studies using the pAkt antibody focused mostly on the cytoplasmic staining, while nuclear phosphorylation of Akt may have more value in determining its effects.

Although we found no significant correlation between pAkt and p53 expression, recent studies have shown that Akt activation promotes $\mathrm{Mdm} 2$ translocation to the nucleus, thereby affecting p53 transcriptional activity and inhibiting p53-dependent apoptosis $(19,20)$.

In this study, we demonstrated that Akt activation was significantly elevated in primary ductal carcinoma with HER2 overexpression. Moreover, it is likely that evaluation of 
pAkt status, especially nuclear pAkt, in addition to hormone receptor status and HER2, will be useful in predicting the efficacy of postoperative endocrine therapy for breast cancer. Our data also suggests that agents which downregulate Akt activation may be of therapeutic value in HER2 overexpressing breast cancers. Moreover, Akt may be a better target than its upstream targets such as HER2, Ras, or PI3K in sensitizing cancer cells to chemotherapy or radiotherapy, because it is located at an important convergent point that may be activated by multiple upstream signal transduction pathways. Our study therefore suggests the clinical benefit of an appropriate combination of conventional chemotherapeutic drugs with new generation signal transduction inhibitors that inhibit the HER2/PI3K/Akt pathway for the treatment of breast cancer.

Nevertheless, to elucidate the significance of Akt activation in clinical outcome, a well-designed prospective study is needed.

\section{References}

1. Nicholson KM and Anderson NG: The protein kinase B/Akt signalling pathway in human malignancy. Cell Signal 14: 381-395, 2002.

2. Jardines L, Weiss M, Fowble B and Greene M: neu(c-erbB2/HER2) and the epidermal growth factor receptor (EGFR) in breast cancer. Pathobiology 61: 268-282, 1993.

3. Earp HS, Dawson TL, Li X and Yu H: Heterodimerization and functional interaction between EGF receptor family members: a new signaling paradigm with implications for breast cancer research. Breast Cancer Res Treat 35: 115-132, 1995.

4. Zhou X, Tan M, Stone Hawthorne V, Klos KS, Lan KH, Yang Y, Yang W, Smith TL, Shi D and Yu D: Activation of the Akt/ mammalian target of rapamycin/4E-BP1 pathway by ErbB2 overexpression predicts tumor progression in breast cancers. Clin Cancer Res 10: 6779-6788, 2004.

5. Clark AS, West K, Streicher S and Dennis PA: Constitutive and inducible Akt activity promotes resistance to chemotherapy, trastuzumab, or tamoxifen in breast cancer cells. Mol Cancer Ther 1: 707-717, 2002.

6. Datta SR, Brunet A and Greenberg ME: Cellular survival: a play in three Akts. Genes Dev 13: 2905-2927, 1999.

7. Thompson JE and Thompson CB: Putting the rap on Akt. J Clin Oncol 22: 4217-4226, 2004.
8. Meier R, Alessi DR, Cron P, Andjelkovic M and Hemmings BA: Mitogenic activation, phosphorylation, and nuclear translocation of protein kinase Bbeta. J Biol Chem 272: 30491-30497, 1997.

9. Andjelkovic M, Alessi DR, Meier R, Fernandez A, Lamb NJ, Frech M, Cron P, Cohen P, Lucocq JM and Hemmings BA: Role of translocation in the activation and function of protein kinase B. J Biol Chem 272: 31515-31524, 1997.

10. Bacus SS, Altomare DA, Lyass L, Chin DM, Farrell MP, Gurova K, Gudkov A and Testa JR: AKT2 is frequently upregulated in HER-2/neu-positive breast cancers and may contribute to tumor aggressiveness by enhancing cell survival. Oncogene 21: 3532-3540, 2002.

11. Perez-Tenorio G and Stal O: Activation of AKT/PKB in breast cancer predicts a worse outcome among endocrine treated patients. Br J Cancer 86: 540-545, 2002.

12. Stal O, Perez-Tenorio G, Akerberg L, Olsson B, Nordenskjold B, Skoog L and Rutqvist LE: Akt kinases in breast cancer and the results of adjuvant therapy. Breast Cancer Res 5: R37-R44, 2003.

13. Ahmad S, Singh N and Glazer RI: Role of AKT1 in 17betaestradiol- and insulin-like growth factor I (IGF-I)-dependent proliferation and prevention of apoptosis in MCF-7 breast carcinoma cells. Biochem Pharmacol 58: 425-430, 1999.

14. Pancholi S, Martin L-A, Lykkesfeldt AE, Dowsett M and Johnston SRD: Upregulation of erbB2/erbB3 and enhanced signal transduction via the phosphatidylinositol 3-kinase pathway in a tamoxifen-resistant breast cancer cell line. Breast Cancer Res Treat 76: S165, 2002.

15. Knuefermann C, Lu Y, Liu B, Jin W, Liang K, Wu L, Schmidt M, Mills GB, Mendelsohn J and Fan Z: HER2/PI-3K/Akt activation leads to a multidrug resistance in human breast adenocarcinoma cells. Oncogene 22: 3205-3212, 2003.

16. Stoica GE, Franke TF, Wellstein A, Czubayko F, List HJ, Reiter R, Morgan E, Martin MB and Stoica A: Estradiol rapidly activates Akt via the ErbB2 signaling pathway. Mol Endocrinol 17: 818-830, 2003.

17. Kirkegaard T, Witton CJ, McGlynn LM, Tovey SM, Dunne B, Lyon A and Bartlett JM: AKT activation predicts outcome in breast cancer patients treated with tamoxifen. J Pathol 207: 139-146, 2005.

18. Schiff R, Massarweh SA, Shou J, Bharwani L, Mohsin SK and Osborne CK: Cross-talk between estrogen receptor and growth factor pathways as a molecular target for overcoming endocrine resistance. Clin Cancer Res 10: 331S-336S, 2004.

19. Zhou BP, Liao Y, Xia W, Zou Y, Spohn B and Hung MC: HER-2/neu induces p53 ubiquitination via Akt-mediated MDM2 phosphorylation. Nat Cell Biol 3: 973-982, 2001.

20. Vestey SB, Sen C, Calder CJ, Perks CM, Pignatelli M and Winters ZE: Activated Akt expression in breast cancer: correlation with p53, Hdm2 and patient outcome. Eur J Cancer 41: 1017-1025, 2005. 